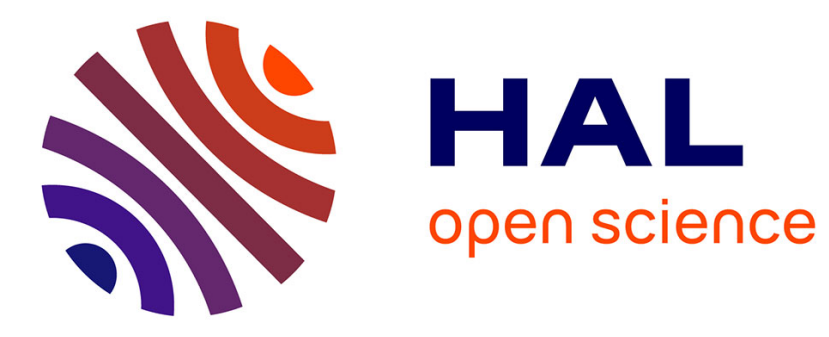

\title{
M2SP: Mining Sequential Patterns Among Several Dimensions
}

\author{
Marc Plantevit, Yeow Wei Choong, Anne Laurent, Dominique Laurent, \\ Maguelonne Teisseire
}

\section{- To cite this version:}

Marc Plantevit, Yeow Wei Choong, Anne Laurent, Dominique Laurent, Maguelonne Teisseire. M2SP: Mining Sequential Patterns Among Several Dimensions. PKDD 2005 - 9th European Conference on Principles and Practice of Knowledge Discovery in Databases, Oct 2005, Porto, Portugal. pp.205-216, 10.1007/11564126_23. lirmm-00106087

\section{HAL Id: lirmm-00106087 https://hal-lirmm.ccsd.cnrs.fr/lirmm-00106087}

Submitted on 18 Nov 2019

HAL is a multi-disciplinary open access archive for the deposit and dissemination of scientific research documents, whether they are published or not. The documents may come from teaching and research institutions in France or abroad, or from public or private research centers.
L'archive ouverte pluridisciplinaire HAL, est destinée au dépôt et à la diffusion de documents scientifiques de niveau recherche, publiés ou non, émanant des établissements d'enseignement et de recherche français ou étrangers, des laboratoires publics ou privés. 


\title{
$M^{2}$ SP: Mining Sequential Patterns among Several Dimensions
}

\author{
M. Plantevit ${ }^{1}$, Y.W. Choong ${ }^{2,3}$, A. Laurent ${ }^{1}$, D. Laurent ${ }^{2}$ and M. Teisseire ${ }^{1}$ \\ 1 LIRMM, Université Montpellier 2, CNRS, 161 rue Ada, 34392 Montpellier, France \\ 2 LICP, Université de Cergy Pontoise, 2 av. Chauvin, 95302 Cergy-Pontoise, France \\ 3 HELP University College, BZ-2 Pusat Bandar Damansara, 50490 Kuala Lumpur, Malaysia
}

\begin{abstract}
Mining sequential patterns aims at discovering correlations between events through time, like A customer who bought a TV and a DVD player at the same time later bought a recorder. Even if many works have dealt with sequential pattern mining, there is no work leading to rules like A customer who bought a surfboard together with a bag in NY later bought a wetsuit in SF. In this paper, we propose $M^{2} S P$, a complete approach to mine such multidimensional sequential patterns. The main originality of our proposition is that we obtain not only intra-pattern sequences but also inter-pattern sequences. Moreover, we consider generalized multidimensional sequential patterns, called jokerized patterns, in which some of the dimension values may not be instanciated. Experiments on synthetic data are reported and show the scalability of our approach.
\end{abstract}

Keywords: Data Mining, Sequential Patterns, Multidimensional Rules.

\section{Introduction}

Mining sequential patterns aim at discovering correlations between events through time. For instance, rules that can be built are A customer who bought a TV and a DVD player at the same time later bought a recorder. Work dealing with this issue in the literature have proposed scalable methods and algorithms to mine such rules [9]. As for association rules, the efficient discovery is based on the support which indicates to which extend data from the database contains the patterns.

However, these methods only consider one dimension to appear in the patterns, which is usually called the product dimension. This dimension may also represent web pages for web usage mining, but there is normally a single dimension. Although some works from various studies claim to combine several dimensions, we argue here that they do not provide a complete framework for multidimensional sequential pattern mining $[4,8,11]$. The way we consider multidimensionality is indeed generalized in the sense that patterns must contain several dimensions combined over time. For instance we aim at building rules like A customer who bought a surfboard and a bag in NY later bought a wetsuit in SF. This rule not only combines two dimensions (City and Product) but it also combines them over time (NY appears before SF, surfboard appears before wetsuit). As far as we know, no method has been proposed to mine such rules. 
In this paper, we present existing methods and their limits from several studies in recent years. We define then the basic concepts associated to our proposition $\mathrm{M}^{2} \mathrm{SP}$ and the algorithms to build such rules. Experiments are performed on synthetic data to assess our proposition.

In our approach, sequential patterns are mined from a relational table, that can be seen as a fact table in a multidimensional database. This is why, contrary to the standard terminology of the relational model, the attributes over which a relational table is defined are called dimensions.

In order to mine such frequent sequences, we extend our approach so as to take into account partially instanciated tuples in sequences. More precisely, our algorithms are designed in order to mine frequent jokerized multidimensional sequences containing as few $*$ as possible, i.e., replacing an occurrence of $*$ with any value from the corresponding domain cannot give a frequent sequence.

This paper is organized as follows. Section 2 introduces a motivating example illustrating the goal of our work. Section 3 presents existing works concerning sequential patterns, multidimensional approaches and multidimensional databases. Section 4 introduces our contribution, $\mathrm{M}^{2} \mathrm{SP}$, as an approach to discover multidimensional sequential patterns. Section 5 introduces jokerized patterns. Section 6 presents the algorithms. Section 7 presents results of experiments performed on synthetic data.

\section{Motivating Example}

In order to illustrate our approach, we consider the following example that will be used throughout the paper as a running example.

Let us consider a relational table $T$ in which transactions issued by customers are stored. More precisely, we assume that $T$ is defined over six dimensions (or attributes) as shown in Fig. 1, and where: $D$ is the date of transactions (considering three dates, denoted by 1,2 and 3), $C G$ is the category of customers (considering two categories, denoted by Educ and Ret, standing for educational and retired customers, respectively), $A$ is the age of customers (considering three discretized values, denoted by $Y$ (young), $M$ (middle) and $O$ (old)), $C$ is the city where transactions have been issued (considering three cities, denoted by $N Y$ (New York), $L A$ (Los Angeles) and $S F$ (San Francisco)), $P$ is the product of the transactions (considering four products, denoted by $c, m, p$ and $r$ ), $Q$ stands for the quantity of products in the transactions (considering nine such quantities).

For instance, the first tuple of $T$ (see Fig. 1) means that, at date 1, educational young customers bought 50 products $c$ in New York. Let us now assume that we want to extract all multidimensional sequences that deal with the age of customers, the products they bought and the corresponding quantities, and that are frequent with respect to the groups of customers and the cities where transactions have been issued. To this end, we consider three sets of dimensions as follows: (i) the dimension $D$, representing the date, (ii) the three dimensions $A, P$ and $Q$ that we call analysis dimensions (tuples over analysis dimensions are those that appear in the items that constitute the sequential patterns to be mined), (iii) the two dimensions $C G$ and $C$, that we call reference dimensions (the table is partitioned according to tuple values over reference dimensions and the support of a given multidimensional sequence is the ratio of the number of blocks supporting 
the sequence over the total number of blocks. Fig. 5 displays the corresponding blocks in our example).

In this framework, $\langle\{(Y, c, 50),(M, p, 2)\},\{M, r, 10)\}\rangle$ is a multidimensional sequence having support $\frac{1}{3}$, since the partition according to the reference dimensions contains 3 blocks, among which one supports the sequence. This is so because $(Y, c, 50)$ and $(M, p, 2)$ both appear at the same date (namely date 1$)$, and $(M, r, 10)$ appears later on (namely at date 2 ) in the first block shown in Figure 4.

It is important to note that, in our approach, more general patterns, called jokerized sequences, can be mined. The reason for this generalization is that considering partially instanciated tuples in sequences implies that more frequent sequences are mined. To see this, considering a support threshold of $\frac{2}{3}$, no sequence of the form $\left\langle\{(Y, c, \mu)\},\left\{\left(M, r, \mu^{\prime}\right)\right\}\right\rangle$ is frequent. On the other hand, in the first two blocks of Fig. 5, $Y$ associated with $c$ and $M$ associated with $r$ appear one after the other, according to the date of transactions. Thus, we consider that the jokerized sequence, denoted by $\langle\{(Y, c, *)\},\{(M, r, *)\}\rangle$, is frequent since its support is equal to $\frac{2}{3}$.

\begin{tabular}{cccccc}
$\begin{array}{c}\text { D } \\
\text { (Date) }\end{array}$ & $\begin{array}{c}\text { CG } \\
\text { (Customer-Group) }\end{array}$ & $\begin{array}{c}\text { C } \\
\text { (City) }\end{array}$ & $\begin{array}{c}\text { A } \\
(\text { Age })\end{array}$ & $\begin{array}{c}\text { P } \\
\text { (Product) }\end{array}$ & $\begin{array}{c}\text { Q } \\
\text { (Quantity) }\end{array}$ \\
\hline 1 & Educ & NY & Y & c & 50 \\
1 & Educ & NY & M & p & 2 \\
1 & Educ & LA & Y & c & 30 \\
1 & Ret. & SF & O & c & 20 \\
1 & Ret. & SF & O & m & 2 \\
2 & Educ & NY & M & p & 3 \\
2 & Educ & NY & M & r & 10 \\
2 & Educ & LA & Y & c & 20 \\
3 & Educ & LA & M & r & 15
\end{tabular}

Fig. 1. Table $T$

\section{Related Work}

\subsection{Sequential Patterns}

An early example of research in the discovering of patterns from sequences of events can be found in [5]. In this work, the idea is the discovery of rules underlying the generation of a given sequence in order to predict a plausible sequence continuation. This idea is then extended to the discovery of finding interesting patterns (or rules) embedded in a database of sequences of sets of events (items). A more formal approach in solving the problem of mining sequential patterns is the AprioriAll algorithm as presented in [6]. Given a database of sequences, where each sequence is a list of transactions ordered by transaction time, and each transaction is a set of items, the goal is to discover all sequential patterns with a user-specified minimum support, where the support of a pattern is the number of data-sequences that contain the pattern. In [1], the authors introduce the problem of mining sequential patterns over large databases of customer transactions where each transaction consists of customer-id, transaction time, and the items bought in the transaction. Formally, given a set of sequences, where each sequence consists of 
a list of elements and each element consists of a set of items, and given a user-specified min support threshold, sequential pattern mining is to find all of the frequent subsequences, i.e., the subsequences whose occurrence frequency in the set of sequences is no less than min support. Sequential pattern mining discovers frequent patterns ordered by time. An example of this type of pattern is A customer who bought a new television 3 months ago, is likely to buy a DVD player now. Subsequently, many studies have introduced various methods in mining sequential patterns (mainly in time-related data) but almost all proposed methods are Apriori-like, i.e., based on the Apriori property which states the fact that any super-pattern of a nonfrequent pattern cannot be frequent. An example using this approach is the GSP algorithm [9].

\subsection{Data to be Mined}

We assume that the reader is familiar with the relational model of databases [10], and we briefly recall the basic ingredients of this model used in this paper. Let $U=\left\{D_{1}, \ldots D_{n}\right\}$ be a set of attributes, which we call dimensions in our approach. Each dimension $D_{i}$ is associated with a (possibly infinite) domain of values, denoted by $\operatorname{dom}\left(D_{i}\right)$. A relational table $T$ over universe $U$ is a finite set of tuples $t=\left\{d_{1}, \ldots, d_{n}\right\}$ such that, for every $i=1, \ldots, n, d_{i} \in \operatorname{dom}\left(D_{i}\right)$. Moreover, given a table $T$ over $U$, for every $i=1, \ldots, n$, we denote by $\operatorname{Dom}_{T}\left(D_{i}\right)$ (or simply $\operatorname{Dom}\left(D_{i}\right)$ if $T$ is clear from the context) the active domain of $D_{i}$ in $T$, i.e., the set of all values of $\operatorname{dom}\left(D_{i}\right)$ that occur in $T$.

Since we are interested in sequential patterns, we assume that $U$ contains at least one dimension whose domain is totally ordered, corresponding to the time dimension.

In our running example, we consider $U=\{D, C G, A, P, Q\}$, where $D$ is the time dimension. Moreover, considering the table $T$ of Fig. 1, we have for instance $\operatorname{Dom}(D)=\{1,2,3\}$ and $\operatorname{Dom}(C G)=\{E d u c$, Ret $\}$.

\subsection{Multidimensional Sequential Patterns}

As far as we know, three propositions have been studied in order to deal with several dimensions when building sequential patterns.

Pinto et al. [8] is the first paper dealing with several dimensions in the framework of sequential patterns. For instance, purchases are not only described by considering the customer ID and the products, but also by considering the age, type of the customer (Cust-Grp) and the city where he lives, as shown in Fig. 1. Multidimensional sequential patterns are defined over the schema $A_{1}, \ldots, A_{m}, S$ where the set of $A_{i}$ stands for the dimensions describing the data and $S$ stands for the sequence of items purchased by the customers ordered over time. A multidimensional sequential pattern is defined as $\left(i d_{1},\left(a_{1}, \ldots, a_{m}\right), s\right)$ where $a_{i} \in A_{i} \cup\{*\} . i d_{1},\left(a_{1}, \ldots, a_{m}\right)$ is said to be a multidimensional pattern. For instance, the authors consider the sequence $((*, N Y, *),\langle b f\rangle)$ meaning that customers from NY have all bought a product $b$ and then a product $f$. Sequential patterns are mined from such multidimensional databases either (i) by mining all frequent sequential patterns over the product dimension and then regrouping them into multidimensional patterns, (ii) or by mining all frequent multidimensional patterns and then mining frequent product sequences over these patterns. Note that the sequences found 
by this approach do not contain several dimensions since the dimension time only concerns products. Dimension product is the only dimension that can be combined over time, meaning that it is not possible to have a rule indicating that when $b$ is bought in Boston then $c$ is bought in $N Y$.

Yu et Chen. [11] considers sequential pattern mining in the framework of Web Usage Mining. Even if they consider three dimensions (pages, sessions, days), these dimensions are very particular since they belong to a single hierarchized dimension. Moreover, the sequences found describe correlations between objects over time by considering only one dimension, which corresponds to the web pages.

de Amo et al. [4] is based on first order temporal logic. This proposition is close to our approach, but there are some limits since (i) groups used to compute the support are predefined whereas we consider the fact that the user should be able to define them (see reference dimensions below), (ii) several attributes cannot appear in the sequences. The authors claim that they aim at considering several dimensions but they have only shown one dimension for the sake of simplicity. However, the paper does not provide any complete proposition to extend this point to real multidimensional patterns.

\section{$4 \quad$ M$^{2}$ SP: Mining Multidimensional Sequential Patterns}

As shown above, no work has been done in order to mine sequential patterns over several dimensions. The work described in [8] is said to be intra-pattern since sequences are mined within the framework of a single description (the so-called pattern). In this paper, we propose to generalize this work to inter-pattern multidimensional sequences.

\subsection{Dimension Partition}

\begin{tabular}{cccccc} 
D & CG & C & A & P & Q \\
\hline 1 & Educ & NY & Y & c & 50 \\
1 & Educ & NY & M & p & 2 \\
2 & Educ & NY & M & p & 3 \\
2 & Educ & NY & M & r & 10
\end{tabular}

Fig. 2. block $(E d u c, N Y)$

\begin{tabular}{cccccc} 
D & CG & C & A & P & Q \\
\hline 1 & Educ & LA & Y & c & 30 \\
2 & Educ & LA & Y & c & 20 \\
3 & Educ & LA & M & r & 15
\end{tabular}

Fig. 3. block $(E d u c, L A)$

\begin{tabular}{cccccc} 
D & CG & C & A & P & Q \\
\hline 1 & Ret. & SF & O & c & 20 \\
1 & Ret. & SF & O & m & 2
\end{tabular}

Fig. 4. block (Ret.,SF)

Fig. 5. blocks defined on $T$ over dimensions $C G$ and C

For each table defined on the set of dimensions $D$, we consider a partition of $D$ into four sets: $D_{t}$ for the temporal dimension, $D_{\mathscr{A}}$ for the analysis dimensions, $D_{\mathscr{R}}$ for the reference dimensions, $D_{\mathscr{F}}$ for the ignored dimensions.

Each tuple $c=\left(d_{1}, \ldots, d_{n}\right)$ can thus be denoted $c=(f, r, a, t)$ with $f$ the restriction on $D_{\mathscr{F}}$ of $c, r$ the restriction on $D_{\mathscr{R}}, a$ the restriction on $D_{\mathscr{A}}, t$ the restriction on $D_{t}$. 
Given a table $T$, the set of all tuples in $T$ having the same value $r$ on $D_{\mathscr{R}}$ is said to be a block and we denote by $\mathscr{B}_{T, D}$ the set of blocks from table $T$. Fig. 5 shows blocks built from table $T$. Each block $B$ in $\mathscr{B}_{T, D_{\mathscr{R}}}$ is denoted by the tuple $r$ that defines it.

In our running example, we consider $\mathscr{F}=\emptyset, D_{\mathscr{R}}=\{C G, C\}, D_{\mathscr{A}}=\{A, P\}, D_{t}=\{D\}$.

When mining multidimensional sequential patterns, the set $D_{\mathscr{R}}$ identifies the blocks of the database to be considered when computing the support. For this reason, this set is called reference. The support of a sequence is the proportion of blocks embedding it. Note that usual sequential patterns, and sequential patterns from [8] and [4], this set is reduced to one dimension (cid in [8] or IdG in [4]). The set $D_{\mathscr{A}}$ describes the analysis dimensions, meaning that these dimensions will be found in the multidimensional sequential patterns. Note that usual sequential patterns only consider one analysis dimension corresponding to the products purchased or the web pages visited. The set $\mathscr{F}$ describes the ignored dimensions, which are used neither to define the date, nor the blocks, and which are not present within the patterns mined.

\subsection{Multidimensional Item, Itemset and Sequential Pattern}

Definition 1 (Multidimensional Item) A multidimensional item e defined on $D_{\mathscr{A}}=$ $\left\{D_{i_{1}}, \ldots, D_{i_{m}}\right\}$ is a tuple $e=\left(d_{i_{1}}, \ldots, d_{i_{m}}\right)$ such that $\forall k \in[1, m], d_{i_{k}} \in \operatorname{Dom}\left(D_{i_{k}}\right)$.

Definition 2 (Multidimensional Itemset) A multidimensional itemset $i$ defined on $D_{\mathscr{A}}=$ $\left\{D_{i_{1}}, \ldots, D_{i_{m}}\right\}$ is a non empty set of items $i=\left\{e_{1}, \ldots, e_{p}\right\}$ where $\forall j \in[1, p], e_{j}$ is a multidimensional item defined on $D_{\mathscr{A}}$ and $\forall j, k \in[1, p], e_{j} \neq e_{k}$.

Note that all items from an itemset are defined using the same dimensions $\left(D_{\mathscr{A}}\right)$. Also note that all pairs of multidimensional items from an itemset are different.

Definition 3 (Multidimensional Sequence) A multidimensional sequence $\zeta$ defined on $D_{\mathscr{A}}=\left\{D_{i_{1}}, \ldots, D_{i_{m}}\right\}$ is an ordered non empty list of itemsets $\varsigma=\left\langle i_{1}, \ldots, i_{l}\right\rangle$ where $\forall j \in$ $[1, l], i_{j}$ is a multidimensional itemset defined on $D_{\mathscr{A}}$.

In our running example, $(Y, c, 50),(M, p, 2),(M, r, 10)$ are three multidimensional items on $D_{\mathscr{A}}=\{A, P, Q\} .\{(Y, c, 50),(M, p, 2)\}$ and $\{(M, r, 10)\}$ are multidimensional itemsets on $D_{\mathscr{A}}$. $\langle\{(Y, c, 50),(M, p, 2)\},\{(M, r, 10)\}\rangle$ is a multidimensional sequence on $D_{\mathscr{A}}$.

Definition 4 (Inclusion of sequence) A multidimensional sequence $\boldsymbol{\varsigma}=\left\langle a_{1}, \ldots, a_{l}\right\rangle$ is said to be a subsequence of a sequence $\zeta^{\prime}=\left\langle b_{1}, \ldots, b_{l^{\prime}}\right\rangle$ if there exist integers $1 \leq j_{1} \leq$ $j_{2} \leq \ldots \leq j_{l} \leq l^{\prime}$ such that $a_{1} \subseteq b_{j_{1}}, a_{2} \subseteq b_{j_{2}}, \ldots, a_{l} \subseteq b_{j_{l}}$.

Let $\varsigma=\langle\{(Y, c, 50)\},\{(M, r, 10)\}\rangle$ and $\varsigma^{\prime}=\langle\{(Y, c, 50),(M, p, 2)\},\{(M, r, 10)\}\rangle$ be two multidimensional sequences. $\zeta$ is a subsequence of $\zeta^{\prime}$.

\subsection{Support}

Computing the support of a sequence amounts to counting the number of blocks that contain the sequence. A block supports a sequence if it is possible to find a set of tuples which satisfy it. For each itemset from the sequence, we must thus find a date from 
$\operatorname{Dom}\left(D_{t}\right)$ such that all items from the itemset appear at this date. All itemsets must be retrieved at different dates from $\operatorname{Dom}\left(D_{t}\right)$ such that the order of the itemsets from the sequence is satisfied.

Definition 5 A table $T$ supports a sequence $\left\langle i_{1}, \ldots, i_{l}\right\rangle$ if $\forall j=1 \ldots l, \exists d_{j} \in \operatorname{Dom}\left(D_{t}\right)$, for every item e in $i_{j}, \exists t=\left(f, r, e, d_{j}\right) \in T$ with $d_{1}<d_{2}<\ldots<d_{l}$.

The block $(E d u c, N Y)$ from Fig. 2 supports $\langle\{(Y, c, 50),(M, p, 2)\},\{(M, r, 10)\}\rangle$ since $\{(Y, c, 50),(M, p, 2)\}$ appears at date $=1$ and $\{(M, r, 10)\}$ appears at date $=2$.

The support of a sequence in a table $T$ is the proportion of blocks of $T$ that support it.

Definition 6 (Sequence Support) Let $D_{\mathscr{R}}$ be the reference dimensions and $T$ be a table partitioned into the set of blocks $\mathscr{B}_{T, D}$. The support of a sequence $\zeta$ is:

$$
\operatorname{support}(\varsigma)=\frac{\mid\left\{B \in \mathscr{B}_{T, D} \text { s.t.B supports } \zeta\right\} \mid}{\left|\mathscr{B}_{T, D}\right|}
$$

Definition 7 (Frequent Sequence) Let minsup $\in[0,1]$ be the minimum user-defined support value. A sequence $\zeta$ is said to be frequent if support $(\varsigma) \geq \operatorname{minsup}$.

Note that an item $e$ is said to be frequent if so is the sequence $\langle\{e\}\rangle$.

Let us consider $D_{\mathscr{R}}=\{C G, C\}, D_{\mathscr{A}}=\{A, P\}$, minsup $=\frac{1}{5}, \varsigma=\langle\{(Y, c, 50),(M, p, 2)\},\{(M, r, 10)\}\rangle$. The three blocks of the partition of $T$ from Fig. 5 must be scanned to compute support $(\varsigma)$.

1. block (Educ, NY) (Fig. 2). Two dates can be found in this block. At date 1, we have $(Y, c, 50)$ and $(M, p, 2)$ and at date 2 , we have $(M, r, 10)$. Thus this block supports $\varsigma$.

2. block (Educ, LA) (Fig. 3). This block cannot be counted since it does not contain $(M, p, 2)$.

3. block (Ret., SF) (Fig. 4). This block contains only one date. Therefore, it is not possible $\varsigma$ to be embedded in it.

We have thus support $(\varsigma)=\frac{1}{3} \geq \operatorname{minsup}$.

\section{Jokerized Sequential Patterns}

Considering the definitions above, an item can only be retrieved if there exists a frequent tuple of values from domains of $D_{\mathscr{A}}$ containing it. For instance, it can occur that neither $(Y, r)$ nor $(M, r)$ nor $(O, r)$ is frequent whereas the value $r$ is frequent. Thus, we introduce the joker value $*$. In this case, we consider $(*, r)$ which is said to be jokerized.

Definition 8 (Jokerized Item) Let $e=\left(d_{1}, \ldots, d_{m}\right)$ a multidimensional item. We denote by $e_{\left[d_{i} / \delta\right]}$ the replacement in e of $d_{i}$ by $\delta$. e is said to be a jokerized multidimensional item if: (i) $\forall i \in[1, m], d_{i} \in \operatorname{Dom}\left(D_{i}\right) \cup\{*\}$, and (ii) $\exists i \in[1, m]$ such that $d_{i} \neq *$, and (iii) $\forall d_{i}=*, \nexists \delta \in \operatorname{Dom}\left(D_{i}\right)$ such that $e_{\left[d_{i} / \delta\right]}$ is frequent.

A jokerized item contains at least one specified analysis dimension. It contains a $*$ only if no specific value from the domain can be set. A jokerized sequence is a sequence containing at least one jokerized item. A block is said to support a sequence if a set of tuples containing the itemsets satisfying the temporal constraints can be found.

Definition 9 (Support of a Jokerized Sequence) A table $T$ supports a jokerized sequence $\varsigma=\left\langle i_{s 1}, \ldots, i_{s l}\right\rangle$ if $\forall j \in[1, l], \exists \delta_{j} \in \operatorname{Dom}\left(D_{t}\right), \forall e=\left(d_{i_{1}}, \ldots, d_{i_{m}}\right) \in i_{j}, \exists t=$ $\left(f, r,\left(x_{i_{1}}, \ldots, x_{i_{m}}\right), \delta_{j}\right) \in T$ with $d_{i}=x_{i}$ or $d_{i}=*$ and $\delta_{1}<\delta_{2}<\ldots<\delta_{l}$.

The support of $\varsigma$ is the proportion of blocks containing $\varsigma$. $\operatorname{support}(\varsigma)=\frac{\mid\left\{B \in \mathscr{B}_{T, D_{\mathscr{R}}} \text { s.t.B supports } \varsigma\right\} \mid}{\left|\mathscr{B}_{T, D_{\mathscr{R}}}\right|}$ 


\section{Algorithms}

\subsection{Mining 1-frequent items}

1 -frequent items are items built on the analysis dimensions. When considering no joker value, a single scan of the database results in the multidimensional items being frequent. When considering jokerized items, a levelwise algorithm is used in order to build the frequent multidimensional items having the smallest possible number of joker values. To this end, we consider a lattice which lower bound is the $(*, \ldots, *)$ multidimensional item. This lattice is partially built from $(*, \ldots, *)$ up to the frequent items containing as few $*$ as possible. At level $i, i$ values are specified. Then items at level $i$ are combined to build a set of candidates at level $i+1$. Two frequent items are combined to build a candidate if they are $\bowtie$-compatible.

Definition $10\left(\bowtie\right.$-compatibility) Let $e_{1}=\left(d_{1}, \ldots, d_{n}\right)$ and $e_{2}=\left(d_{1}^{\prime}, \ldots, d_{n}^{\prime}\right)$ be two distinct multidimensional items where $d_{i}$ and $d_{i}^{\prime} \in \operatorname{dom}\left(D_{i}\right) \cup\{*\}$. $e_{1}$ and $e_{2}$ are said to be $\bowtie$-compatible if $\exists \Delta=\left\{D_{i_{1}}, \ldots, D_{i_{n-2}}\right\} \subset\left\{D_{1}, \ldots, D_{n}\right\}$ such that for every $j \in[1, n-2]$, $d_{i_{j}}=d_{i_{j}}^{\prime} \neq *$ with $d_{i_{n-1}}=*$ and $d_{i_{n-1}}^{\prime} \neq *$ and $d_{i_{n}} \neq *$ and $d_{i_{n}}^{\prime}=*$.

Definition 11 (Join) Let $e_{1}=\left(d_{1}, \ldots, d_{n}\right)$ and $e_{2}=\left(d_{1}^{\prime}, \ldots, d_{n}^{\prime}\right)$ be two $\bowtie$-compatible multidimensional items. We define $e_{1} \bowtie e_{2}=\left(v_{1}, \ldots, v_{n}\right)$ where $v_{i}=d_{i}$ if $d_{i}=d_{i}^{\prime}, v_{i}=d_{i}$ if $d_{i}^{\prime}=*$ and $v_{i}=d_{i}^{\prime}$ if $d_{i}=*$.

Let $E$ and $E^{\prime}$ be two sets of multidimensional items of size n, we define

$E \bowtie E^{\prime}=\left\{e \bowtie e^{\prime}\right.$ s.t. $\left(e, e^{\prime}\right) \in E \times E^{\prime} \wedge e$ and $e^{\prime}$ are $\bowtie$-compatible $\}$

Two multidimensional items defined on $n$ dimensions are $\bowtie$-compatible if they share $n$ 2 values. For instance, $(N Y, Y, *)$ and $(*, Y, r)$ are $\bowtie$-compatible. We have $(N Y, Y, *) \bowtie$ $(*, Y, r)=(N Y, Y, r)$. On the contrary, $(N Y, M, *)$ and $(N Y, Y, *)$ are not $\bowtie$-compatible. Note that this method is close to the one used for iceberg cubes in $[2,3]$.

$F_{1}^{i}$ stands for the set of 1-frequent items having $i$ dimensions which are specified (different from $*$ ). Frequent items of size 1 are obtained from the candidate items of size 1. More generally, the algorithm to build candidate items of size $i$ are obtained by considering the frequent items of size $i-1: F_{1}^{1}=\left\{f \in \operatorname{Cand}_{1}^{1}\right.$, support $\left.(f) \geq \operatorname{minsup}\right\}$, Cand $_{1}^{i}=F_{1}^{i-1} \bowtie F_{1}^{i-1}$.

\subsection{Mining Jokerized Multidimensional Sequences}

Once 1-frequent items are mined, the candidate sequences of size $k(k \geq 2)$ are generated and validated to keep the frequent items. This computation is based on usual algorithms such as PSP [7] by introducing the treatment of joker values.

The support of a sequence $\zeta$ in a table $T$ according to the reference dimensions $D_{\mathscr{R}}$ is given by Algorithm 1. The reference dimensions are used to compute the partition to be considered. This algorithm checks whether each block of the partition supports the sequence by calling the function supportTable (Algorithm 2). supportTable attempts to find a tuple from the block that matches the first item of the first itemset of the sequence in order to anchor the sequence. This operation is repeated recursively until all itemsets from the sequence are found (return true) or until there is no way to go on further (return false). Several possible anchors may be tested if the first ones do not fit. 


\section{Function supportcount}

Data $: \varsigma, T, D_{\mathscr{R}}$, counting

Result : support of $\varsigma$

Integer support $\longleftarrow 0$; BooleanseqSupported;

$\mathscr{B}_{T, D_{\mathscr{R}}} \longleftarrow$ blocks of $T$ identified over $\left.D_{\mathscr{R}}\right\}$;

foreach $B \in \mathscr{B}_{T, D_{\mathscr{R}}}$ do

seqSupported $\longleftarrow$ supportTable ( $\varsigma, B$, counting) ;

if seqSupported then support $\longleftarrow$ support +1 ;

return $\left(\frac{\text { support }}{\left|\mathscr{B}_{T, D}\right|}\right)$

Algorithm 1: Support of a sequence (supportcount)

\section{Function supportTable}

Data : $\varsigma, T$, counting //counting indicates whether joker values are considered or not Result : Boolean

ItemSetFound $\longleftarrow$ false; seq $\longleftarrow$; itset $\longleftarrow$ sequence.first () ; it $\longleftarrow$ itset first ()

if $\varsigma=\emptyset$ then return (true) // End of Recursivity

while $t \longleftarrow T$.next $\neq \emptyset$ do

if supports $(t$, it, counting) then

if $($ NextItem $\longleftarrow-$ itset.second ()$)=\emptyset$ then ItemSet Found $\longleftarrow$ true

// Look for all the items from the itemset

else

// Anchoring on the item (date)

$T^{\prime} \longleftarrow \sigma_{\text {date }=t \text {.date }}(T)$

while $t^{\prime} \longleftarrow T^{\prime}$.next ()$\neq \emptyset \wedge$ ItemSetFound $=$ false do

if supports $\left(t^{\prime}\right.$, NextItem, counting $)$ then NextItem « itset.next ()

if NextItem $=\emptyset$ then ItemSetFound $\longleftarrow$ true

if ItemSetFound = true then

// Look now for the other itemsets

return (supportTable(seq.tail ()$, \sigma_{\text {date }>\text { t.date }}(T)$, counting $)$ )

else

itset $\longleftarrow$ seq.first ()

$C \longleftarrow \sigma_{\text {date }>\text { t.date }}(T) / /$ Skip to next dates

return(false) // Not found

Algorithm 2: supportTable (Checks if a sequence $\varsigma$ is supported by a table $T$ ) 


\section{Experiments}

In this section, we report experiments performed on synthetic data. These experiments aim at showing the interest and scalability of our approach, especially in the jokerized approach. As many databases from the real world include quantitative information, we have distinguished a quantitative dimension. In order to highlight the particular role of this quantitative dimension, we consider four ways of computing frequent sequential patterns: (i) no joker $\left(M^{2} S P\right.$ ), (ii) jokers on all dimensions but the quantitative one $\left(M^{2} S P\right.$-alpha), (iii) jokers only on the quantitative dimension $\left(M^{2} S P-m u\right)$, (iv) jokers on all dimensions $\left(M^{2} S P\right.$-alpha-mu). Note that case (iv) corresponds to the jokerized approach presented in Section 5. Our experiments can thus be seen as being conducted in the context of a fact table of a multidimensional database, where the quantitative dimension is the measure. In Figures 5-12, minsup is the minimum support taken into account, nb_dim is the number of analysis dimensions being considered, DB_size is the number of tuples, avg_card is the average number of values in the domains of the analysis dimensions.

Fig. 6 and 7 compare the behavior of the four approaches described above when the support changes. $M^{2} S P$-alpha and $M^{2} S P$-alpha-mu have a similar behavior, the difference being due to the verification of quantities in the case of $M^{2} S P$-alpha. Note that these experiments are not led on the same minimum support values since no frequent items are found for $M^{2} S P$ and $M^{2} S P$-mu if the support is too high. Fig. 8 shows the scalability of our approach since runtime grows almost linearly when the database size increases (from 1,000 tuples up to 26,000 tuples). Fig. 9 shows how runtime behaves when the average cardinality of the domains analysis dimensions changes. When this average is very low, numerous frequent items are mined among few candidates. On the contrary, when this average is high, numerous candidates have to be considered from which few frequent items are mined. Between these two extrema, the runtime decreases. Fig. 10 and 11 show the behavior of our approach when the number of analysis dimensions changes. The number of frequent items increases as the number of analysis dimensions grows, leading to an increase of the number of frequent sequences. Fig. 12 and 13 show the differential between the number of frequent sequences mined by our approach compared to the number of frequent sequences mined by the approach described in [8], highlighting the interest of our proposition.

\section{Conclusion}

In this paper, we propose a novel definition for multidimensional sequential patterns. Contrary to the propositions from [4,8,11], several analysis dimensions can be found in the sequence. This allows the discovery of rules like A customer who bought a surfboard together with a bag in NY later bought a wetsuit in LA. We also define jokerized sequential patterns by introducing the joker value $*$ on analysis dimensions. Moreover, reference dimensions are defined in order to generalize the way clients are defined. The algorithms implementing our approach are given and evaluated with experiments performed on synthetic data.

This work can be extended following several directions. For example, we can take into account approximate values on quantitative dimensions. In this case, we allow 

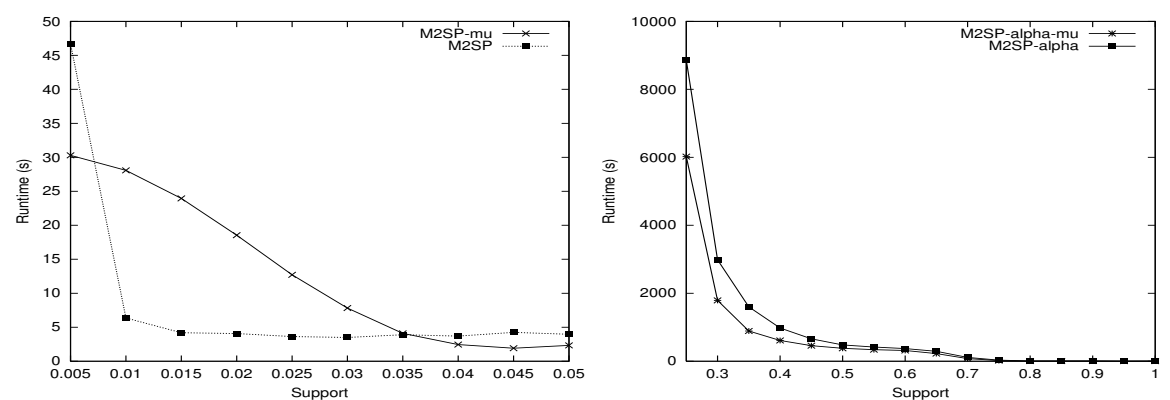

Fig. 6. Runtime over Support (DB_size $=12000$, nb_dim=5, avg_card=20)

Fig. 7. Runtime over Support (DB_size $=12000$, nb_dim $=5$, avg_card=20)
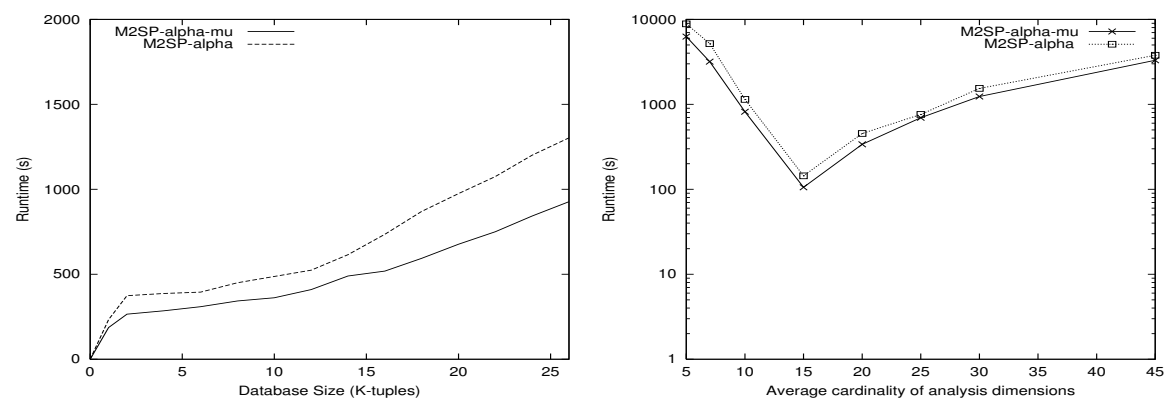

Fig. 8. Runtime over database size (minsup $=0.5, \quad$ nb_dim $=15$, avg_card $=$ 20)

Fig. 9. Runtime over Average Cardinality of Analysis Dimensions (minsup $=0.8$, DB_size=12000, nb_dim=15)
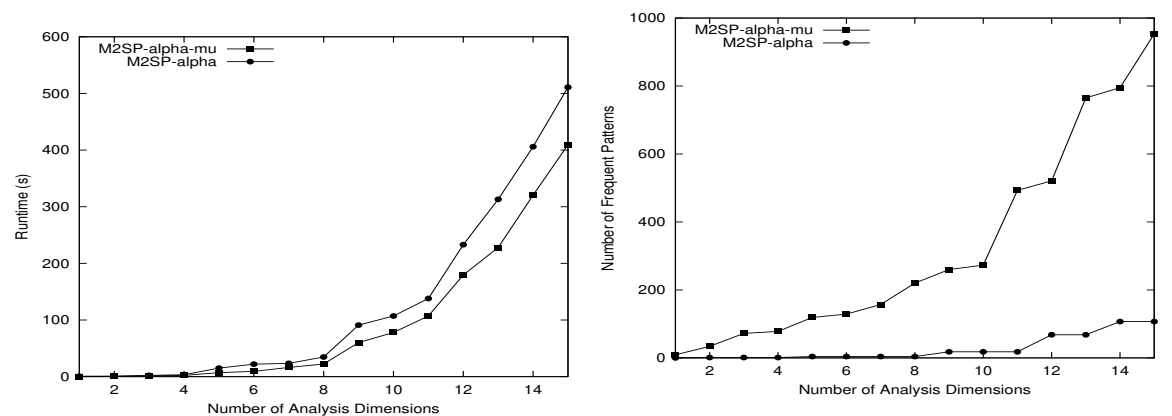

Fig. 10. Runtime over Number of Analysis Dimensions (minsup $=0.5$, DB_size $=12000$, nb_dim $=15$, avg_card=20)

Fig. 11. Number of Frequent patterns over number of analysis dimensions (minsup=0.5, DB_size $=12000$, nb_dim $=15$, avg_card=20) 


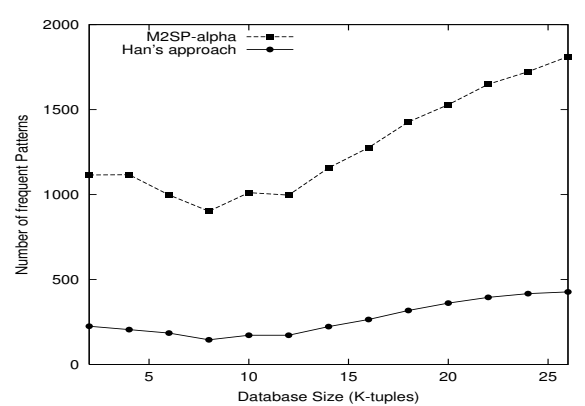

Fig. 12. Number of Frequent Sequences over Database Size (minsup $=0.5$, nb_dim=15, avg_card=20)

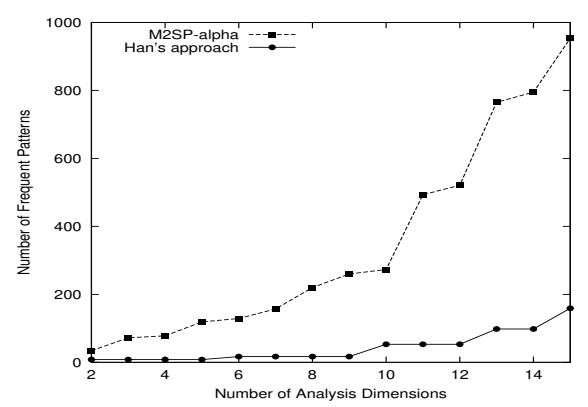

Fig. 13. Number of Frequent Sequences over Number of Analysis Dimensions (minsup $=0.5, \quad$ DB_size $=12000$, avg_card=20)

the consideration of values that are not fully jokerized while remaining frequent. This proposition is important when considering data from the real world where the high number of quantitative values prevent each of them to be frequent. Rules to be built will then be like The customer who bought a DVD player on the web is likely to buy almost $3 D V D s$ in a supermarket later. Hierarchies can also be considered in order to mine multidimensional sequential patterns at different levels of granularity in the framework of multidimensional databases.

\section{References}

1. R. Agrawal and R. Srikant. Mining sequential patterns. In Proc. 1995 Int. Conf. Data Engineering (ICDE'95), pages 3-14, 1995.

2. K. Beyer and R. Ramakrishnan. Bottom-up computation of sparse and iceberg cube. In Proc. of ACM SIGMOD Int. Conf. on Management of Data, pages 359-370, 1999.

3. A. Casali, R. Cicchetti, and L. Lakhal. Cube lattices: A framework for multidimensional data mining. In Proc. of 3rd SIAM Int. Conf. on Data Mining, 2003.

4. S. de Amo, D. A. Furtado, A. Giacometti, and D. Laurent. An apriori-based approach for first-order temporal pattern mining. In Simpósio Brasileiro de Bancos de Dados, 2004.

5. T.G. Dietterich and R.S. Michalski. Discovering patterns in sequences of events. Artificial Intelligence, 25(2):187-232, 1985.

6. H. Mannila, H. Toivonen, and A.I. Verkamo. Discovering frequent episodes in sequences. In Proc. of Int. Conf. on Knowledge Discovery and Data Mining, pages 210-215, 1995.

7. F. Masseglia, F. Cathala, and P. Poncelet. The PSP Approach for Mining Sequential Patterns. In Proc. of PKDD, volume 1510 of LNCS, pages 176-184, 1998.

8. H. Pinto, J. Han, J. Pei, K. Wang, Q. Chen, and U. Dayal. Multi-dimensional sequential pattern mining. In ACM CIKM, pages 81-88, 2001.

9. R. Srikant and R. Agrawal. Mining Sequential Patterns: Generalizations and Performance Improvements. In Proc. of EDBT, pages 3-17, 1996.

10. J.D. Ullman. Principles of Database and Knowledge-Base Systems, volume I. Computer Science Press, 1988.

11. C.-C. Yu and Y.-L. Chen. Mining sequential patterns from multidimensional sequence data. IEEE Transactions on Knowledge and Data Engineering, 17(1):136-140, 2005. 